\title{
Formulation Development and Evaluation of Venlafaxine HCL Buccal Patch
}

\author{
Anand G. Mundhe ${ }^{1, *}$, Gokul A. Khairnar ${ }^{2}$, Sadik F. Sayyad ${ }^{3}$ \\ ${ }^{1}$ Dept. of Pharmaceutics S.M.B.T College of Pharmacy, Nandi Hills, Dhamangaon, Tal. Igatpuri, Dist. Nasik (M.S) -422 403 \\ ${ }^{2}$ Department of Pharmaceutical Technology, University Institute of Chemical Technology, North Maharashtra University, \\ Jalgaon, 425 001, Maharashtra \\ ${ }^{3}$ Dept. of pharmaceutics Amrutvahini College of pharmacy Sagamner, Ahmadnagar, Maharashtra \\ *Corresponding Author: anandmundhe@gmail.com
}

Copyright (C) 2014 Horizon Research Publishing All rights reserved.

\begin{abstract}
Within the oral mucosal cavity, the buccal region offers an attractive route of administration for Systemic patches. Venlafaxine were Prepared using HPMC K4M, Pectin, and poly vinyl Pyrrolidone, PG as Plasticizers. FTIR and UV spectroscopic and DSC methods revealed that there is no interaction between Venlafaxine and polymers. The patches were evaluated for their thickness uniformity, folding endurance, Weight Uniformity, content uniformity, Swelling behaviour, tensile strength, Mucoadhesion strength and Surface $\mathrm{pH}$. In vitro release studies of Venlafaxine loaded patches in Phosphate buffer (pH, 6.8) exhibited $98 \%$ drug release in the $8 \mathrm{~h}$. The optimized patch was obtained good in vitro result.
\end{abstract}

Keywords Bioadhesion, Buccal Patch, Pectin, Venlafaxine HCL, HPMC K4M, Drug Release

\section{Introduction}

Buccal delivery of drug provides an attractive alternative to the oral route of drug administration. Buccal drug delivery highly effective ways to improve bioavailability because of high blood supply, drug directly enter in to systemic circulation $[1,2]$. Buccal drug delivery offers a safer method of drug delivery, since drug action can be promptly terminated in case of toxicity by removing the dosage from the buccal cavity [3]. The adhesive properties of such drug delivery platforms can reduce the enzymatic degradation due to the increased intimacy between the delivery vehicle and the absorbing membrane [4]. It is also possible to administer drugs to patients who cannot be given drugs orally for one reason or other $[5,6]$. Drug with short half-life, requiring sustained or controlled release showing poor aqueous solubility and which is sensitive to enzymatic degradation, may be successfully delivered across the buccal mucosa [7]. Buccal patches are of two types (a) matrix type and (b) reservoir type. In matrix type system (3), the drug is homogeneously dispersed in hydrophilic or lipophilic polymer matrix and the medicated polymer is then molded into medicated disc with a defined surface area. In this system, the adhesive polymer is spread along the circumference to form a strip of adhesive rim around the medicated disc. In reservoir type systems, the drug core is encapsulated by rate controlling polymeric membrane [8-10]. Venlafaxine HCL (VNLF) is an antidepressant of the serotonin-nor epinephrine reuptake inhibitor (SNRI) class first introduced by Wyeth in 1993. It is prescribed for the treatment of clinical depression and anxiety disorders. VNLF is well absorbed. Bioavailability is $45 \%$ following oral administration. The degree of binding of VNLF to human plasma is $27 \% \pm 2 \%$ at concentrations ranging from 2.5 to $2215 \mathrm{ng} / \mathrm{mL}$. Plasma half life is $5 \mathrm{~h}$. Undergoes extensive first pass metabolism in the liver to its major, active metabolite, ODV, and two minor, less active metabolites, $\mathrm{N}$-desmethylvenlafaxine and $\mathrm{N}$, O-didesmethylvenlafaxine [11]. The main aim of the present work was to prepare buccal patch of Venlafaxine in order to reduce the first pass metabolisam and improve its oral bioavailability by using combination of two polymers i.e. (pectin and HPMC) in a suitable solvent system.

\section{Materials and Methods}

\subsection{Materials}

VNLF was received as a gift sample from Meditab Daman India; Pectin was a purchase from Loba Chemical Mumbai India. HPMC K4M was obtained from S.D. fine chemical Mumbai, PVP K30 was received from BASF Mumbai. All other reagents and Chemicals were of analytical grade and were used as such..

\subsection{Methods}

2.2.1. Preparation of mucoadhesive buccal patches[10] 
Buccal patches containing drug reservoir was prepared by solvent casting methods. PVP was used as mucoadhesive polymer and propylene glycol $(30-50 \%$ of the polymer weight) as plasticizer. Acetic acid $1 \% \mathrm{v} / \mathrm{v}$ solution was prepared in which weighed quantity of HPMC was properly dispersed. Then weighed quantity of pectin was taken and mixed with HPMC solution to make final mixture. PVP was accurately weighed and mix in pectin solution. The required quantity of plasticizer propylene glycol was added. Then the drug was dispersed uniformly in the viscous solution with continuous stirring. The resultant mixture was poured into specially fabricated Petri dish $(5 \times 5 \mathrm{~cm})$ lined with aluminum foil. Drying was carried out at room temperature for 24 hours. The drying rate was controlled by placing an inverted glass funnel. This arrangement also controlled the effect of current on the films. For complete drying, the Petri dish was kept in a hot-air oven maintained at $45 \pm 1^{\circ} \mathrm{C}$ for another 12 hours. After complete drying, the films were removed from the Petri dish. The films were smooth, flexible and could be cut to any desired size and shape.

Table 1. Formula Composition

\begin{tabular}{|c|c|c|c|c|c|c|c|c|}
\hline Batch & F1 & F2 & F3 & F4 & F5 & F6 & F7 & F8 \\
\hline VNLF & 100 & 100 & 100 & 100 & 100 & 100 & 100 & 100 \\
\hline HPMC & 184 & 92 & 184 & 92 & 184 & 92 & 92 & 184 \\
\hline Pectin & 184 & 92 & 184 & 184 & 92 & 92 & 184 & 92 \\
\hline PVP-k30 & 80 & 40 & 40 & 80 & 80 & 80 & 40 & 40 \\
\hline
\end{tabular}

*Propylene glycol (30-50\% of the polymer weight) as plasticizer. * Acetic acid $(1 \% \mathrm{v} / \mathrm{v})(\mathrm{ml})$ are used as solvent

\section{Evaluation of Buccal Patch}

\section{Mass Uniformity and Thickness}

Mass uniformity of the patches was studied in 10 different randomly selected patches from each batch. Thickness was measured by the screw gauge [11].

\section{Folding Endurance}

Folding endurance of patches was determined manually by repeatedly folding a films at the same place until it ruptures. The number of folding required to break or crack a patch was taken as the folding endurance [12].

\section{Drug Content Uniformity}

Drug content uniformity was determined by dissolving the patch by homogenization in $100 \mathrm{ml}$ of an isotonic phosphate buffer $(\mathrm{pH}$ 6.8) for $2 \mathrm{~h}$ with constant shaking and $5 \mathrm{ml}$ samples was withdrawn and diluted with isotonic phosphate buffer $\mathrm{pH} 6.8$ up to $20 \mathrm{ml}$, and this solution was filtered through a $0.45 \mathrm{~mm}$ Whatman filter paper. The drug content was then determined spectrofluorometerically at $224 \mathrm{~nm}$ [13].

\section{Surface Ph Determination}

The patches were allowed to swell by keeping them in contact with $1 \mathrm{ml}$ of distilled water for $2 \mathrm{~h}$ at room temperature, and $\mathrm{pH}$ was noted down by bringing the electrode in contact with the surface of the patch, allowing it to equilibrate for $1 \mathrm{~min}$ [14].

\section{In Vitro Mucoadhesion}

The mucoadhesive strength of patches was measured in triplicate on a modified physical balance. A piece of sheep buccal mucosa was tied to the mouth of a glass vial filled completely with PBS pH 6.8. The glass vial was tightly fitted in the center of a beaker filled with PBS at $37^{\circ} \mathrm{C}$. Patches were stuck to the lower side of rubber stoppers with glue and the mass $(\mathrm{g})$ required to detach the patches from the mucosal surface was taken as the mucoadhesive strength (shear stress) [15]. The following parameters were calculated from the mucoadhesive strength force of adhension $(\mathrm{N})=($ mucoadhesive strength $(\mathrm{g})) / 1000 \times 9.81$

\section{In Vitro Permeability}

The mucosal permeation of Venlafaxine through sheep buccal mucosa was determined using a modified Franz diffusion cell (Fig. 2). Briefly, the receptor compartment (17 $\mathrm{mL}$ ) was filled with PBS (pH 6.8) at constant stirred staring. The patch was placed between the donor and receptor compartments of the diffusion cell on the sheep buccal mucosa. Aliquots $(2 \mathrm{~mL})$ of the receptor medium were withdrawn at regular intervals and replaced immediately with equal volumes of PBS ( $\mathrm{pH}$ 6.8). The amount of Venlafaxine released into the receptor medium was determined by measurement of absorption at $224 \mathrm{~nm}$ against a blank $[9,15]$.

\section{In Vitro Release Studies}

In vitro drug release studies was determined by using dissolution test apparatus type II (USP) paddle method using $200 \mathrm{ml}$ of phosphate buffer (pH 6.8) as the dissolution medium at $50 \mathrm{rpm}$ at $37 \pm 0.5 \mathrm{C}$ for 8 To provide unidirectional release, one side of each patch was attached to a glass disk with the help of adhesive [15].

\section{Swelling Studies}

The degree of swelling of mucoadhesive polymer is an important factor affecting adhesion. The swelling rate of mucoadhesive patch was evaluated by placing the patch in phosphate buffer solution $\mathrm{pH} 6.8$ at $37 \pm 1 \mathrm{C}$. Six patches of each batch were cut and weighed, and the average weight was calculated (W 1 ). The patches were placed in phosphate buffer and were removed at time intervals of $0.1,2$ 
excess water on the surface was carefully absorbed using filter paper, and swollen patches were reweighed. The average weight $\mathrm{W} 2$ was calculated, and the swelling index was calculated by the formula: $[9,14]$.

$$
\text { S.I. }=(\mathrm{Wt}-\mathrm{W} 0) / \mathrm{W} 0 \times 100
$$

\section{FTIR Study}

The possible interaction between drug and polymers were assessed using Fourier transform infrared spectroscopy (FTIR), model Shimadzu FTIR 8400. FTIR spectra were obtained at room temperature, about $2 \mathrm{mg}$ of pure drug, polymers and formulations were dispersed in $\mathrm{KBr}$ powder and the pellets were made by applying $6000 \mathrm{~kg} / \mathrm{cm} 2$ pressure. FT-IR spectra were obtained by powder diffuse reflectance on FT-IR spectrometer.

\section{DSC Analysis}

This was also carried out to find possible interaction between drug and polymer this was performed on pure drug and drug loaded polymer by using the DSC.

\section{Result and Discussion}

\section{Evaluation of Buccal Patch}

The results of evaluation parameters for the buccal patch revealed that there was no variations in weight of patch as all patch were found to be within the range limit for weight variation and good elasticity and flexibility. The thickness was found to be 0.3 to $0.5 \mathrm{~mm}$. Folding Endurance of F5 high because higher amount of HPMC.To increasing concentration of HPMC to increase folding endurance. The surface $\mathrm{pH}$ is in range of 6.5 to 7.11 closes as neutral there is no risk of the irritation.

\section{Drug Content}

Drug contents of buccal patch from each batch were determined by UV Spectrophotometric method at wavelength $224 \mathrm{~nm}$. The results showed drug content in the range of $95 \%$ to $98 \%$ which was within the acceptable Pharmacopoeial limits,

\section{Swelling Studies}

The degree of swelling was determined in phosphate buffer $\mathrm{pH}$ 6.8. All batches have good swelling properties which remain hydrated for longer time. All formulations were swelled within $10 \mathrm{~min}$ and which delayed the swelling after $2 \mathrm{~h}$ i.e. constant weight of the buccal patch was seen. It was highlighted that swelling properties are important when film integrity is evaluated. HPMC have an increased swelling capacity. F5 formulation showed high swelling index because of the high concentration of Pvp k-30, Hpmc and pectin combination ratio 2:1.there is no effect of plasticizers on swelling index.

\section{Mucoadhesion Strength}

The In-vitro mucoadhesion strength of the buccal patch was determined using goat buccal mucosa. The mucoadhesive strength of patches was measured in the triplicate manner on modified physical balance. Peak detachment force is the maximum applied force at which the film detaches from tissue. The mucoadhesivity of buccal patch was found to be maximum in case of formulation F5 i.e. (pectin and Hpmc ratio 1:2).This may due to fact that positive charges on surface of pectin could give strong electrostatic interaction with mucous or negatively charged mucous membrane.

\section{In- Vitro Permeability}

The mucosal permeation of Venlafaxine through sheep buccal mucosa was determined using a modified Franz diffusion cell. Briefly, the receptor compartment $(17 \mathrm{~mL})$ was filled with PBS (pH 6.8) at constant stirred staring. The patch was placed between the donor and receptor compartments of the diffusion cell on the sheep buccal mucosa. The permeability showed f5 formulation after $8 \mathrm{hr}$ $83.4 \%$.

Table 2. Evaluation of buccal patches

\begin{tabular}{|c|c|c|c|c|c|c|}
\hline Batch code & $\begin{array}{c}\text { Thickness } \\
(\mathrm{mm})\end{array}$ & Mass $(\mathrm{mg})$ & Drug content & $\begin{array}{c}\text { Folding } \\
\text { Endurance } \\
\text { (times) }\end{array}$ & Force of adhesion $(\mathrm{N})$ & Surface $\mathrm{pH}$ \\
\hline F1 & $0.55 \pm 0.01$ & $88 \pm 1.5$ & $98 \pm 2$ & $140 \pm 5$ & $0.29 \pm 0.3$ & $7.1 \pm 0.11$ \\
\hline F2 & $0.45 \pm 0.01$ & $49 \pm 1$ & $96 \pm 1$ & $155 \pm 4$ & $0.25 \pm 0.2$ & $7.0 \pm 0.13$ \\
\hline F3 & $0.43 \pm 0.02$ & $84 \pm 0.5$ & $97 \pm 2$ & $139 \pm 6$ & $0.28 \pm 0.2$ & $6.8 \pm 0.12$ \\
\hline F4 & $0.34 \pm 0.05$ & $70 \pm 6$ & $97 \pm 3$ & $160 \pm 2$ & $0.24 \pm 0.1$ & $6.5 \pm 0.08$ \\
\hline F5 & $0.42 \pm 0.02$ & $70 \pm 0.5$ & $97 \pm 1$ & $189 \pm 7$ & $0.24 \pm 0.5$ & $7.2 \pm 0.16$ \\
\hline F6 & $0.45 \pm 0.01$ & $53 \pm 0.5$ & $95 \pm 2$ & $175 \pm 3$ & $0.26 \pm 0.1$ & $6.7 \pm 0.14$ \\
\hline F7 & $0.34 \pm 0.05$ & $66 \pm 0.5$ & $98 \pm 2$ & $167 \pm 4$ & $0.27 \pm 0.6$ & $7.1 \pm 0.10$ \\
\hline F8 & $0.47 \pm 0.05$ & $66 \pm 1.1$ & $96 \pm 1$ & $156 \pm 5$ & $0.17 \pm 0.12$ & $6.9 \pm 0.13$ \\
\hline
\end{tabular}




\section{In-Vitro Release Study}

To determine whether the availability of Venlafaxine HCL was increased by formulating the buccal patch, in-vitro drug dissolution studies were carried out in phosphate buffer $(\mathrm{pH}$ 6.8) using USP dissolution test apparatus. In vitro releases were carried out using USP paddle dissolution Type apparatus. The medium used diffusion study phosphate buffer $\mathrm{pH} 6.8$ and vitro release profile from F-5 batch showed maximum release $98.77 \%$ of drug within $8 \mathrm{hr}$

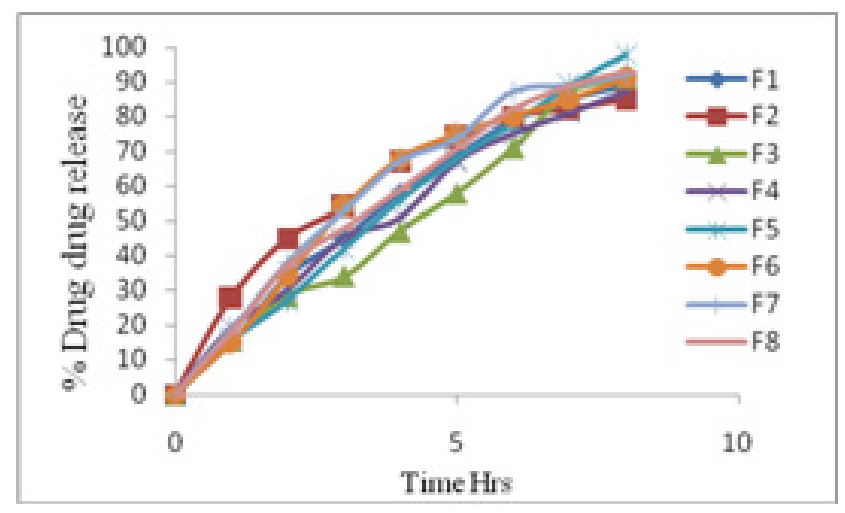

Figure 1. in vitro drug release profile

\section{Correlation Study}

The permeation studies were conducted using Franz diffusion cell assembly. The study was carried out on batch $\mathrm{F}$ 5 which showed $98.06 \%$. Good correlation between Vitro drug release and Vitro permeation, as correlation coefficient was found

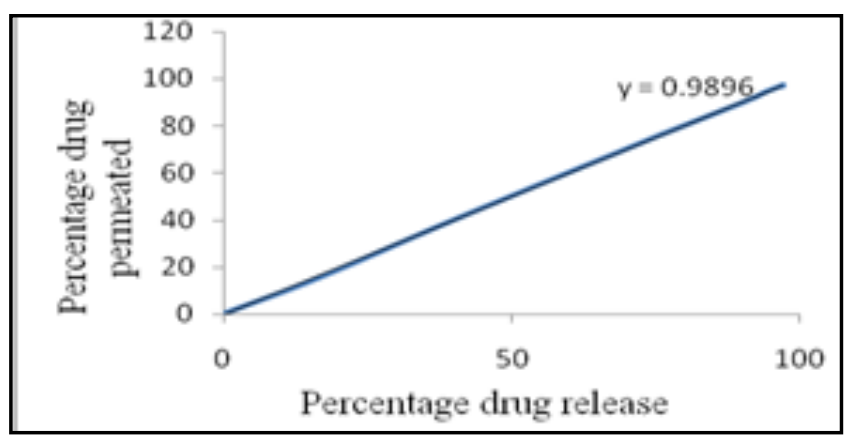

Figure 2. Correlation study

\section{Dissolution Kinetics}

The dissolution kinetics of optimized batch was applied to various dissolution models such as Zero order, First order, and Higuchi, Korsmeyer-Peppas and Hixon crowell. The best fitted model gives the highest R2 value and least slope value. Thus, zero order fits best for the dissolution data of the best fit batch as it showed the highest value for R2 indicates that drug released by diffusion mechanism. The formulation indicates that the drug release continues and constant until drug at absorption site.
Table 3. Kinetic models

\begin{tabular}{|c|c|c|c|}
\hline Sr. No. & Models & $\mathrm{R}^{2}$ & $\mathrm{~K}$ \\
\hline 1 & Zero order & 0.9953 & 12.69 \\
\hline 2 & First order & 0.8718 & 0.74 \\
\hline 3 & Higuchi & 0.9454 & 29.81 \\
\hline 4 & Koresmeyer-Peppas & 0.9867 & 13.024 \\
\hline 5 & Hixon Crowell & 0.9526 & 0.16 \\
\hline
\end{tabular}

\section{FTIR Analysis}

To study the compatibility of drug with excipient IR spectra of drug in combination with excipient in 1:10 ratio was studied. The IR spectrum shown in indicates that there was no physicochemical interaction in between drug and the used excipient. Results of the Preformulation study suggest that all the studied excipient were compatible with Venlafaxine HCL. The spectra obtained FTIR spectroscopy studied at wavelength $3500^{-\mathrm{cm}}$ to $400^{-\mathrm{cm}}$

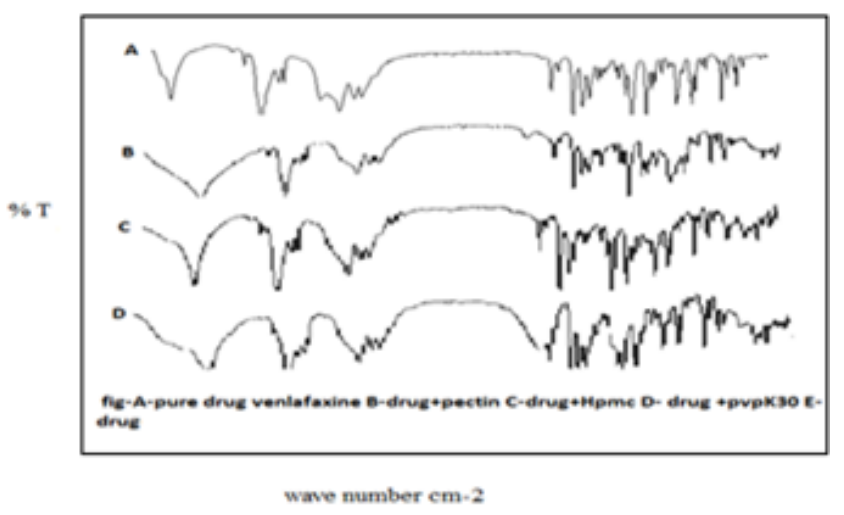

Figure 3. FTIR of pure drug and excipient

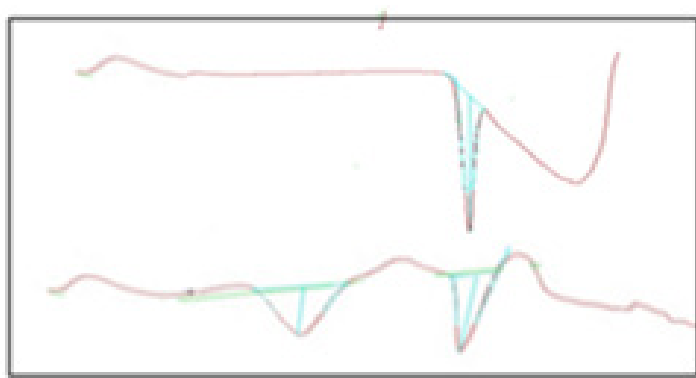

Temp

Time (min)

Figure 4. DSC of Venlafaxine and polymer combination

\section{DSC Analysis}

This was also carried out to find possible interaction between drug and polymer this was performed pure drug and drug loaded polymer by using the DSC. The thermo gram was obtained by. The thermo gram was obtained by. DSC thermo gram of Venlafaxine shows the sharp endothermic peak at $214{ }^{\circ} \mathrm{C}$ due to melting of a drug. The Venlafaxine reported melting point of Venlafaxine HCL $215{ }^{\circ} \mathrm{C}$ and 
observed melting point of Venlafaxine HCL was observed $214^{\circ} \mathrm{C}$ and drug and polymer mixture melting point was observed $215^{\circ} \mathrm{C}$ there is no changes melting point. Above observation suggested that no interaction between drug and polymer.

\section{Conclusion}

From the present investigation can be conclude that the optimized mucoadhesive patches of Venlafaxine hydrochloride with combination of pectin, HPMC, PVP K-30 can meet ideal requirement for buccal device, which can good way to bypass or avoids the extensive hepatic first pass metabolisms and F1 showed highest mucoadhesive strength because pectin to forming secondary bond with mucin and interpenetration polymeric chain with mucin. F5 showed highest swelling index as well as drug release because of high HPMC as well as PVP K-30 content.

\section{Acknowledgment}

Authors would like to thank Wockhardt Research Center, Aurangabad (M.S.) India for providing free sample of drug.

\section{Declaration of Interest}

No conflict of interest.

\section{REFERENCES}

[1] Hoogstraate A J, Verhoef J C, Tuk B, Pijpers A, van Leengoed L A. In vivo buccal delivery of fluorescein isothiocyanate-dextran 4400 with glycodeoxycholate as an absorption enhancer in pigs. J Pharm Sci.(1996) 85:457-60.

[2] Patel V M, Prajapati B G, Patel M M. Design and characterization of chitosan-containing mucoadhesive buccal patches of propranolol hydrochloride. Acta Pharm (2007) $57: 61-72$.
[3] Bussmann W D, Dries R R, Wagner W. Controlled release nitro-glycerine in buccal and oral form. Advances in pharmacotherapy. (1982) 17-25.

[4] Owens T S, Dansereau R J, Sark A. Development and evaluation of extended release bioadhesive sodium fluoride tablets. Int J Pharm. (2005) 288:109-22.

[5] Ishida M, Nambu N, Nagai T. Highly viscous gel ointment containing Carbopol for application to the oral mucosa.Chem.Pharm Bull (1983) 31:4561-4564.

[6] Packer M, Coats A J, Fowler M B, Katus H A, Krum H, Mohacsi P. Effect of Carvedilol on survival in severe chronic heart failure. J Med (2001) 344:1651-1658.

[7] Anders R, Merkle HP. Evaluation of laminated mucoadhesive patches for buccal drug delivery. Int J Pharm (1989) 49:231-240.

[8] Guo J H. Investigating the surface properties and bioadhesion of buccal patches. J Pharm Pharmacol (1994) 46:647-50.

[9] Khairnar G A, Sayyad F J. Development of buccal Drug delivery system based on mucoadhesive polymers, International Journal of PharmTech Research.(2010)2(1): 719-735

[10] Patel V M, Prajapati B G, Patel M M. Effect of hydrophilic polymers on muco-adhesive Eudragit patches of propranolol hydrochloride using factorial design. AAPS Pharm SciTech (2007) 8

[11] Sandeep D, Tiwari A K. Design and evaluation of buccal patches of lornoxicam international Journal of pharma and Bio sciences. (2010)1(4)

[12] Kaur A, Kaur G. Mucoadhesive buccal patches based on inter- polymer complexes of chitosan-pectin for delivery of carvedilol. Saudi Pharm J (2012) 20: 21-27.

[13] Nafee N A, Boraie M A, Ismail F A, Motada L M. Design and characterization of mucoadhesive buccal patches containing cetylpyridinium chloride. Acta Pharm (2003) 3:199-212.

[14] Prasanth V.V, Mamatha Y, Selvi Arunkumar1, Sam T Mathew Abin Abraham. Formulation and Evaluation of Mucoadhesive Buccal Patches of Aceclofenac. Der Pharmacia Lettre.(2012) 4 (1):297-306

[15] Bhanja Satyabrata, P Ellaiah, Rohit Choudhury, KVR Murthy Panigrahi Bibhutibhusan, Martha Sujit Kumar. Design and evaluation of methotrexate buccal mucoadhesive patches. Int J Pharm Biomed Sci (2010) 1(2): 31-36 